\title{
Concordance between the Mini-Mental State Examination, Short Portable Mental Status Questionnaire and Montreal Cognitive Assessment Tests for Screening for Cognitive Impairment in Older Adults
}

\author{
F. Campos-Vasquez ${ }^{a}$, N. Valdez-Murrugarra ${ }^{a}$, A. Soto-Tarazona ${ }^{a}$, K. Camacho-Caballero ${ }^{b, d}, *$, \\ M. A. Rodriguez-Cuba ${ }^{b, d}$, J. F. Parodi ${ }^{c}$, and F. M. Runzer-Colmenares ${ }^{b, c, d, * *}$ \\ ${ }^{a}$ Universidad Peruana de Ciencias Aplicadas, Lima, 15023 Perú \\ ${ }^{b}$ Facultad de Ciencias de la Salud, Carrera de Medicina Humana, \\ Universidad Científica del Sur, Lima, 15067 Perú \\ ${ }^{c}$ Universidad de San Martín de Porres, Facultad de Medicina Humana, \\ Centro de Investigación del Envejecimiento (CIEN), Lima, 15009 Perú \\ ${ }^{d}$ CHANGE Research Group, Carrera de Medicina Humana, Universidad Científica del Sur, Lima, 15067 Perú \\ *e-mail:100014121@ucientifica.edu.pe \\ **e-mail:frunzer@cientifica.edu.pe \\ Received January 12, 2021; revised January 12, 2021; accepted March 16, 2021
}

\begin{abstract}
Determine the level of concordance between the Mini-Mental State Examination (MMSE), Short Portable Mental State Examination (SPMSQ), and Montreal Cognitive Assessment (MoCA) screening test for cognitive impairment in older adults. A cross-sectional study based on an original cohort study. 1683 patients over 60 years-old were included between 2010 and 2015. Demographic information was collected and the MMSE, MoCA, and SPMSQ scores were obtained. Categorical variables were presented as frequencies and percentages, while numerical ones as median and interquartile range. The agreement was measured and adjusted by the number of years of education by Cohen's Kappa index $(k)$ with a 95\% confidence interval (CI). The agreement was considered as good if $k>0.80$. MMSE classified $43.32 \%$ of the patients as having cognitive impairment, MoCA 43.14\%, and SPMSQ 24.84\%. MMSE and MoCA showed an agreement $(k)$ of 0.99 with a 95\% CI of $0.99-1.00$; MoCA and SPMSQ showed a $k$ of 0.43 (95\% CI: $0.38-0.46$ ). Finally, MMSE and SPMSQ showed a $k$ of 0.42 (95\% CI: 0.37-0.46). The results did not change when performing the analysis by education subgroups. There was a strong concordance between MoCA and MMSE tests. Nevertheless, the SPMSQ was discordant with the other tests.
\end{abstract}

Keywords: cognitive dysfunction, brief cognitive test, cognitive screening, elderly, geriatrics

DOI: $10.1134 /$ S2079057021030036

\section{INTRODUCTION}

Within occidental societies, older adults proportion have increased due to the population aging process, conditioned by the increase of life expectancy [1]. In Perú, $82.3 \%$ of the female elderly population has a chronic disease compared to $72.9 \%$ of males [2]. Among chronic diseases, cognitive impairment following dementia places a significant physical, emotional, and financial burden. It is considered a clinical syndrome that frustrates the capacity to perform independently and $39-50 \%$ of the diagnosis is overlooked affecting global public health [3, 4].

Cognitive impairment can be assessed through different tests. The Mini-Mental State Examination (MMSE) evaluate different areas such as orientation, memory, attention, calculation, and language. This is the most popular test to measure cognitive impairment $[5,6]$. Cut-off scores for the diagnosis are set at 24-26 or less out of a maximum of 30 points [7]. Short Portable Mental Status Questionnaire (SPMSQ) evaluates short and long-term memory, orientation in the environment, information on current events, and the ability to perform mathematical tasks. A total score of 5 or more is considered abnormal [8-10].

Finally, a test that assesses the mild and early cognitive impairment is the Montreal Cognitive Assessment (MoCA) that also appraises cognitive areas as attention and concentration, executive functions, memory, language, visuospatial dexterity, conceptual reasoning, calculation, and orientation. Cognitive impairment is considered under 25 points being the highest score of 30 points [11]. 
Table 1. Sociodemographic characteristics and cognitive impairment screening test in Peruvian elderly patients, $n=1683$

\begin{tabular}{l|c}
\hline \multicolumn{1}{c|}{ Characteristics } & $n, \%$ \\
\hline Sex & $687(40.82)$ \\
$\quad$ memale & $996(59.18)$ \\
\hline male & $78(74-84)$ \\
Age in years* & $24.4(22.2-28.1)$ \\
Living status & $232(13.78)$ \\
Weight loss & $563(33.45)$ \\
Polipharmacy & $556(33.47)$ \\
\hline Civil status & \\
single & $46(2.73)$ \\
married & $1205(71.60)$ \\
widower/divorced cohabiting & $309(18.36)$ \\
cohabiting & $62(3.68)$ \\
\hline Education & \\
less than 11 years & $447(26.56)$ \\
more or equal than 11 years & $1236(73.44)$ \\
\hline Comorbidities & $327(19.72)$ \\
chronic kidney disease & $288(17.37)$ \\
type 2 diabetes & $107(6.36)$ \\
congestive heart failure & $1023(61.04)$ \\
hypertension & $181(10.75)$ \\
hypothyroidism & $356(21.39)$ \\
chronic pulmonary disease & $26(1-28)$ \\
\hline Mini Mental State Examination (MMSE)* & $2(1-3)$ \\
\hline Short Portable Mental Status Questionnarie (SPMSQ)* & $27(22-28)$ \\
\hline Montreal Cognitive Assessment (MoCA)* & \\
\hline Median and Interquartile Range (IQR). Sociodemographic characteristics in elderly patients included in the study from Naval \\
Medical Center. & \\
\hline
\end{tabular}

To date, some studies have compared MoCA and MMSE scores and shown that the MoCA test is more sensible and specific than MMSE to detect mild cognitive impairment. It is also easier to conduct and consider as an effective screening tool [12-14].

According to Helmi et al., MoCA and MMSE tests are similar regarding the assessment of general cognitive function with an emphasis on neuropsychological aspects [15]. However, vis a vis comparison of these three tools have not been evaluated in Latin American countries.

We aimed to evaluate the concordance between the MMSE, SPMSQ, and MoCA screening test for cognitive impairment in older adults in a cohort of Peruvian outpatients.

\section{EXPERIMENTAL}

This was a cross-sectional study that involved a total of 1683 participants based on a secondary analy- sis from a published cohort study "Prevalence and Factors Associated with Frailty Among Peruvian Older Adults" [16]. Subjects were recruited from the Geriatrics Service of the Peruvian Navy Medical Center (Centro Médico Naval "Cirujano Mayor Santiago Tavara") (CEMENA) between 2010 and 2015. All participants were over 60 years old and resided in Lima or Callao, Peru, most of them men retired from military service. Patients without complete MMSE, MoCA o SPMSQ scores were excluded from the study $(n=214)$.

Demographic information was collected including age, sex, body mass index (BMI), living status, loss of weight, polypharmacy, civil status, number of years of education, and comorbidities including chronic kidney disease (CKD), type-2 diabetes mellitus (T2DM), heart failure (HF), hypertension, hypothyroidism, and chronic obstructive pulmonary disease (COPD). Scores were obtained at the beginning of the study (visit zero). There were missing data in the study due to non-response for some conditions. 25 participants 
Table 2. Kappa index of screening tests for cognitive impairment in elderly patients, $n=1683$

\begin{tabular}{|c|c|c|c|}
\hline \multirow{2}{*}{\multicolumn{2}{|c|}{ Screening test }} & \multicolumn{2}{|c|}{ MMSE } \\
\hline & & \multirow{3}{*}{$\begin{array}{c}\text { cognitive impairment } \\
726 \\
3\end{array}$} & \multirow{3}{*}{$\begin{array}{c}\text { no cognitive impairment } \\
0 \\
954\end{array}$} \\
\hline \multirow{2}{*}{$\mathrm{MoCA}$} & Cognitive impairment & & \\
\hline & No cognitive impairment & & \\
\hline \multicolumn{4}{|c|}{ Kappa Index (CI 95\%): $0.999(0.992-1.000), p<0.001$} \\
\hline \multirow{2}{*}{\multicolumn{2}{|c|}{ Screening test }} & \multicolumn{2}{|c|}{ MMSE } \\
\hline & & cognitive impairment & no cognitive impairment \\
\hline \multirow{2}{*}{ SPMSQ } & Cognitive impairment & 346 & 72 \\
\hline & No cognitive impairment & 383 & 882 \\
\hline \multicolumn{4}{|c|}{ Kappa Index (CI 95\%): $0.421(0.379-0.462), p<0.001$} \\
\hline \multirow{2}{*}{\multicolumn{2}{|c|}{ Screening test }} & \multicolumn{2}{|c|}{$\mathrm{MoCA}$} \\
\hline & & cognitive impairment & no cognitive impairment \\
\hline \multirow{2}{*}{ SPMSQ } & Cognitive impairment & 346 & 72 \\
\hline & no cognitive impairment & 380 & 885 \\
\hline
\end{tabular}

Kappa Index (CI 95\%): 0.423 (0.382-0.464), $p<0.001$

* CI 95\%-Confidence Interval 95\%; MMSE-Mini Mental State Examination; SPMSQ-Short Portable Mental Status Questionnaire; MoCA-Montreal Cognitive Assessment. Kappa Index from cognitive impairment screening test in elderly patients included in the study from Naval Medical Center.

did not answer about the presence or not of CKD and T2DM. Also, 19, 14 and 22 participants respectively did not answer about the presence or not of COPD, weight loss, and polypharmacy, corresponding as missing data too.

To determine cognitive impairment, a score greater than 4 on the SPMSQ, less than 26 on MoCA, and less than 25 in MMSE were taken as a cutoff point. Categorical variables were presented as frequencies and percentages, while numerical as median and interquartile range. The agreement between MMSE, MoCA, and SPMSQ was measured by Cohen's Kappa index. The agreement is represented by the Kappa coefficient $(k)$ and is considered optimal if $k>0.80$. Results were presented in a two-way contingency table of frequencies. The kappa Cohen's index was sub-calculated in subgroups divided according to the number of years of education of the participants (over 11 years of education received). A $p$-value of less than 0.05 was considered statistically significant. Statistical analysis was performed using STATA 14.0 (REF).

The study was approved by the research ethics committee of the Peruvian Navy Medical Center and Universidad Peruana de Ciencias Aplicadas (UPC).

\section{RESULTS AND DISCUSSION}

A total of 1683 patients were evaluated, $59.18 \%$ were men and $74.29 \%$ were married. $73 \%$ of the patients received 11 or more years of education. The most prevalent comorbidities were: hypertension
(61.04\%), osteoarthritis (47.65\%), and COPD $(21.39 \%)$. The prevalence of cognitive impairment according to MMSE, MoCA, and SPMSQ was 43.32, $43.14,24.84 \%$, respectively. The subject's sociodemographic characteristics are shown in Table 1.

As shown in Table 2 MMSE and MoCA showed the highest agreement resulting in a $k$ of $0.99,95 \% \mathrm{CI}$ (0.99-1.00). Concordance between MoCA and SPMSQ resulted in an index of 0.43 with a $95 \%$ CI of $0.38-0.46$. The major disagreement was between MMSE and SPMSQ, resulting in an index of 0.42 with a $95 \%$ CI of $0.37-0.46$. The kappa index and their 95\% CIs from the three analyzes do not vary significantly when performing the analysis by education subgroups; these data are shown in Table 3.

Despite being a global public health problem, cognitive impairment is an overlooked diagnosis in 35$90 \%$ [4]. MMSE and MoCA are commonly used in clinical practice as an assessment of mild cognitive impairment (MCI).

We found an excellent correlation between the MoCA and MMSE tests. The very good agreement between these two tests coincides with similar studies performed in other countries but different populations [17-19]. Given that it has been described that the MMSE test is dependent on education, an analysis adjusted by years of study was performed, resulting in an adequate agreement.

In Peru, the MMSE test has been previously validated [20], as there is an excellent agreement with the MoCA screening test, it could help the healthcare pro- 
Table 3. Kappa index of screening tests scores for cognitive impairment in elderly patients by number of years of education, $n=1683$

\begin{tabular}{l|c|c|c|c|c|c}
\hline \multirow{2}{*}{ Screening test } & \multicolumn{3}{|c|}{$\begin{array}{c}\text { Subgroup: more than 11 years } \\
\text { of education, } n=1236\end{array}$} & \multicolumn{3}{c}{ Subgroup: less than 11 years of education, } \\
& \multicolumn{2}{|c|}{$n=447$} & \\
\cline { 2 - 7 } & kappa index & CI 95\% & $p$-value & kappa index & CI 95\% & $p$-value \\
\hline MMSE vs. MOCA & 0.999 & $0.989-1.000$ & $<0.001$ & 1.00 & $1.00-1.0$ & $<0.001$ \\
MMSE vs. SPMSQ & 0.413 & $0.363-0.462$ & $<0.001$ & 0.439 & $0.366-0.517$ & $<0.001$ \\
MOCA vs. SPMSQ & 0.416 & $0.367-0.466$ & $<0.001$ & 0.442 & $0.366-0.517$ & $<0.001$ \\
\hline
\end{tabular}

Kappa index for cognitive impairment screening test scores from elderly patients included in the study divided in subgroups by number of years of education (cut-point 11 years), from the Naval Medical Center "Cirujano Mayor Santiago Távara," 2010-2015.

fessional to decide between applying any of these two tests to determine cognitive impairment. The importance of this result is highlighted since in our environment low-cost tests are necessary due to inequity in access to evaluation by a neuropsychiatrist or psychiatrist. Therefore, using these tests in the first level of care, adequate screening could be achieved for cognitive impairment or MCI when applied in a similar population [21].

We evaluated SPMSQ as a potential screening tool given its simplicity. However, the agreement was suboptimal when compared with MMSE or MoCA. This can be explained because the tests are different from each other since they evaluate different areas of higher brain functions [22]. Based on our results, this tool cannot be recommended as an initial test to determine cognitive impairment at the time of the comprehensive geriatric assessment. However, recent bibliography mentions its possible use for risk stratification in geriatric patients in the emergency setting [23].

Certain limitations should be mentioned. A secondary database was used and included retired navy members and their immediate relatives who generally have a higher level of education and a lifestyle, so the findings could not necessarily be extrapolated to other groups of geriatric populations. Also, the scales that have been used in this research do not evaluate dementia but assess cognitive impairment.

\section{CONCLUSIONS}

There was an excellent agreement between MMSE and MoCA and both tests can be used as a tool for assessment of cognitive impairment. SPMSQ cannot be recommended for the initial screening of cognitive impairment in the elderly.

Compliance with Ethical Standards. The authors declare that they have no conflict of interest.

\section{COMPLIANCE WITH ETHICAL STANDARDS}

Conflict of interests. The authors declare that they have no conflicts of interest.
Statement on the welfare of humans or animals. All applicable international, national, and/or institutional guidelines for the care and use of animals were followed.

\section{REFERENCES}

1. Ezquiaga, E., García-López, A., Montes, J.M., et al., Discapacidad en pacientes bipolares ancianos en tratamiento ambulatorio. Variables asociadas, Rev. Psiquiatr. Salud Mental, 2012, vol. 5, no. 3, pp. 183-190. https://doi.org/10.1016/j.rpsm.2011.04.004

2. Situación de la Población Adulta Mayor: Abril-MayoJunio 2020, Lima: Inst. Nac. Estadistica Inf., 2020, no. 3.

3. Duong, S., Patel, T., and Chang, F., Dementia: What pharmacists need to know, Can. Pharm. J., 2017, vol. 150 , no. 2 , pp. $118-129$. https://doi.org/10.1177/1715163517690745

4. Valcour, V.G., Masaki, K.H., Curb, J.D., and Blanchette, P.L., The detection of dementia in the primary care setting, Arch. Intern. Med., 2000, vol. 160, no. 19, pp. 2964-2968.

https://doi.org/10.1001/archinte.160.19.2964

5. Creavin, S.T., Wisniewski, S., Noel-Storr, A.H., et al., Mini-Mental State Examination (MMSE) for the detection of dementia in clinically unevaluated people aged 65 and over in community and primary care populations, Cochrane Database Syst. Rev., 2016, vol. 1. https://doi.org/10.1002/14651858.CD011145.pub2

6. Castillo, M.J.M. and Romero, A.C., Detección del deterioro cognitivo en los pacientes mayores de 50 años ingresados Hospital Roberto Calderón Gutiérrez-Managua-Nicaragua, Rev. Cienc. Salud Educ. Med., 2017, vol. 1, no. 1, pp. 9-15. https://revistacienciasmedicas.unan.edu.ni/index.php/rcsem/article/view/15.

7. Scheffels, J.F., Fröhlich, L., Kalbe, E., and Kessler, J., Concordance of Mini-Mental State Examination, Montreal Cognitive Assessment and Parkinson Neuropsychometric Dementia Assessment in the classification of cognitive performance in Parkinson's disease, $J$. Neurol. Sci., 2020, vol. 412, p. 116735.

https://doi.org/10.1016/j.jns.2020.116735

8. Pfeiffer, E., A short portable mental status questionnaire for the assessment of organic brain deficit in elderly patients, J. Am. Geriatr. Soc., 1975, vol. 23, no. 10, pp. 433-441.

9. Erkinjuntti, T., Sulkava, R., Wikström, J., and Autio, L., Short Portable Mental Status Questionnaire as a screen- 
ing test for dementia and delirium among the elderly, $J$. Am. Geriatr. Soc., 1987, vol. 35, no. 5, pp. 412-416. https://doi.org/10.1111/j.1532-5415.1987.tb04662.x

10. Urrunaga-Pastor, D., Moncada-Mapelli, E., RunzerColmenares, F.M., et al., Factors associated with poor balance ability in older adults of nine high-altitude communities, Arch. Gerontol. Geriatr., 2018, vol. 77, pp. 108-114. https://doi.org/10.1016/j.archger.2018.04.013

11. Loureiro, C., García, C., Adana, L., et al., Uso del test de evaluación cognitiva de Montreal (MoCA) en América Latina: revisión sistemática, Rev. Neurol., 2018, vol. 66, pp. 397-408. https://doi.org/10.33588/rn.6612.2017508

12. Delgado, C., Araneda, A., and Behrens, M.I., Validación del instrumento Montreal Cognitive Assessment en español en adultos mayores de 60 años, Neurologia, 2019, vol. 34, no. 6, pp. 376-385. https://doi.org/10.1016/j.nrl.2017.01.013

13. Fisekovic, S., Memic, A., and Pasalic, A., Correlation between MoCA and MMSE for the assessment of cognition in schizophrenia, Acta Inf. Med., 2012, vol. 20, no. 3, pp. 186-189. https://doi.org/10.5455/aim.2012.20.186-189

14. van Steenoven, I., Aarsland, D., Hurtig, H., et al., Conversion between mini-mental state examination, Montreal cognitive assessment, and dementia rating scale-2 scores in Parkinson's disease, Mov. Disord., 2014, vol. 29, no. 14, pp. 1809-1815. https://doi.org/10.1002/mds.26062

15. Helmi, L., Meagher, D., O’Mahony, E., et al., Agreement and conversion formula between mini-mental state examination and Montreal cognitive assessment in an outpatient sample, World J. Psychiatry, 2016, vol. 6 , no. 3 , p. 358 . https://doi.org/10.5498/wjp.v6.i3.358

16. Runzer-Colmenares, F.M., Samper-Ternent, R., Al Snih, S., et al., Prevalence and factors associated with frailty among Peruvian older adults, Arch. Gerontol. Geriatr., 2014, vol. 58, no. 1, pp. 69-73. https://doi.org/10.1016/j.archger.2013.07.005
17. Roalf, D.R., Moore, T.M., Mechanic-Hamilton, D., et al., Bridging cognitive screening tests in neurologic disorders: a crosswalk between the short Montreal Cognitive Assessment and Mini-Mental State Examination, Alzheimer's Dementia, 2017, vol. 13, no. 8, pp. 947-952.

https://doi.org/10.1016/j.jalz.2017.01.015

18. Cao, L., Hai, S., Lin, X., et al., Comparison of the Saint Louis University Mental Status Examination, the Mini-Mental State Examination, and the Montreal Cognitive Assessment in detection of cognitive impairment in Chinese elderly from the geriatric department, J. Am. Med. Directors Assoc., 2012, vol. 13, no. 7, pp. 626-629.

https://doi.org/10.1016/j.jamda.2012.05.003

19. Larner, A., Correlation or limits of agreement? Applying the Bland-Altman approach to the comparison of cognitive screening instruments, Dementia Geriatr. Cognit. Disord., 2016, vol. 42, nos. 5-6, pp. 247-254. https://doi.org/10.1159/000450701

20. Custodio, N. and Lira, D., Adaptación peruana del Minimental State Examination (MMSE), An. Fac. Med., 2014, vol. 75, no. 1, p. 69. https://doi.org/10.15381/anales.v75i1.6951

21. Nasreddine, Z.S., Phillips, N.A., Bédirian, V., et al., The Montreal Cognitive Assessment, MoCA: a brief screening tool for mild cognitive impairment, J. Am. Geriatr. Soc., 2005, vol. 53, no. 4, pp. 695-699. https://doi.org/10.1111/j.1532-5415.2005.53221.x

22. Pinedo, L., Jimeno, H.C., Morales, A.H., et al., Valoración geriátrica integral en adultos mayores hospitalizados a nivel nacional, Diagnostico, 2004, vol. 43, no. 2. http://www.fihu.org.pe/revista/numeros/2004/ mar-abr04/57-63.html.

23. Moyer, V.A., Screening for cognitive impairment in older adults: US Preventive Services Task Force recommendation statement, Ann. Intern. Med., 2014, vol. 160, no. 11 , pp. $791-797$. https://doi.org/10.7326/M14-0496 